\title{
LOS ESTUDIOS SOBRE JUVENTUD EN CHILE: COORDENADAS PARA UN ESTADO DEL ARTE*
}

\author{
OSCAR AGUILERA RUIZ ${ }^{* *}$
}

\begin{abstract}
RESUMEN
La producción académica sobre la juventud en Chile es cada vez mayor, aunque ella no se encuentre enmarcada necesariamente en un campo de estudios institucionalizado, con debates permanentes entre los investigadores y con difusión pública de sus trabajos. De allí que en el presente artículo se proponga un conjunto de coordenadas iniciales que permitan construir un Estado del Arte sobre la materia, como paso fundamental en la evaluación del conocimiento producido y las posibilidades de legitimidad e institucionalización académica y política de esta área transdisciplinar de estudio que define la preocupación por lo juvenil.

PAlABRAS ClaVe: JUVENTUd, ChILE, ESTAdo DEL ARTE, ENFOQUES

* Artículo elaborado sobre la base de la tesis doctoral realizada por el autor (Aguilera, 2008).

** Doctor en Antropología Social y Cultural. Académico del Departamento de Sociología de la Universidad Católica Cardenal Silva Henríquez. E-Mail: oscar.aguilera@gmail.com.
\end{abstract}




\title{
OS ESTUDOS SOBRE JUVENTUDE NO CHILE: COORDENADAS PARA UM ESTADO DA ARTE
}

\begin{abstract}
RESUMO
A produção acadêmica sobre a juventude no Chile é cada vez maior, ainda que não se encontre inscrita necessariamente em um campo de estudos institucionalizado, com debates permanentes entre os pesquisadores e com difusão pública de seus trabalhos. O presente artigo propõe um conjunto de coordenadas iniciais que permita a construção de um Estado da Arte sobre o tema. Este é um passo fundamental na avaliação do conhecimento produzido e as possibilidades de legitimidade e institucionalização acadêmica e política desta área transdisciplinar de estudo que define a preocupação com o juvenil.

PALAVRAS-CHAVE: JUVENTUde, ChILE, ESTADO DA ARTE, ENFOQUES

\section{STUDIES ON CHILEAN YOUTH: COORDINATED FOR A STATE OF ART}

Abstract

The academic production on youth in Chile is growing, although it is not necessarily framed in an institutionalized field of study, with ongoing discussions between researchers and public dissemination of its work. Hence in this article proposes a set of initial coordinates that can bring about a state of the art on the subject, as a key step in evaluating the knowledge produced and the potential legitimacy and institutionalization of academic and disciplinary policy in this area of study which defines the concern to youth.
\end{abstract}

KEY WORDS: YOUTH, CHILE, STATE OF ART, APPROACHES 


\section{INTRODUCCIÓN}

LA PREOCUPACIÓN SOBRE LA juventud en la literatura académica de las ciencias sociales y humanas puede ser fijada en distintos periodos del siglo XX si asumimos como punto de vista el desarrollo e institucionalización de las disciplinas; así podríamos señalar los trabajos desarrollados por los sociólogos de Chicago que en el marco de los procesos de transformación sociocultural de los años 20 en EE.UU. se ocupan de comprender las modalidades de sociabilidad que desarrollan, principalmente, la primera generación de norteamericanos hijos de migrantes europeos (Trasher 1926, Whyte 1942) y que constituyen trabajos que intentan contrarrestar las visiones psicoevolutivas presentadas por Hall (1904), y que presenta a los y las jóvenes como sujetos dominados por sus impulsos y carentes de maduración para enfrentar racionalmente la vida.

Si recurrimos a una entrada desde la disciplina antropológica podríamos señalar los pioneros trabajos de Margaret Mead en los años 20 y que intenta demostrar bajo los preceptos boasianos la enorme variabilidad de formas culturales de vivir la juventud y la sexualidad y por esa vía impugnar los postulados universalistas provenientes fundamentalmente de la matriz psicológica. O reconocer el aporte al estudio que realiza Jean Monod en los años 60 para analizar la conformación de bandas juveniles a partir de su emergencia y apropiación de los espacios públicos en el París de la época, así como la tradición institucionalizada de estudios en juventud en España (Romani, 1989; Feixa, 1998).

Sería injusto no considerar el trabajo de los sociólogos de la cultura que desde Birmingham (Hall y Jefferson, 1983) recuperan los conceptos gramscianos de ideología, subalternidad y hegemonía, para estudiar las prácticas y rituales de resistencia que desarrollan los jóvenes británicos de la posguerra y el surgimiento de subculturas juveniles espectaculares que han marcado el desarrollo de los estilos juveniles a nivel mundial y que poseen plena vigencia como son los punks (Hedbige, 2003) y los hippies (Hall, 1977).

$\mathrm{O}$, finalmente, no considerar la tradición investigativa latinoamericana que desde comienzos de los años 80' viene desarrollando conocimiento y teorización sobre las juventudes del continente y que ha permitido contar con acabados estudios de los chavos banda en México (Urteaga, 2000; Reguillo, 1995; Valenzuela, 2002), los movimientos estudiantiles en Argentina (Balardini, 2002), el análisis de los jóvenes como movimiento social (Faletto, 1986). 
Sostengo que el hilo invisible que une a todos y cada uno de estos puntos de partida, con independencia de los enfoques, es la conceptualización implícita de la juventud como una categoría política; el conjunto de trabajos dedicados a la juventud y a los que hemos referido anteriormente de modo ilustrativo se inscriben en el marco de desarrollo e institucionalización de los Estados nacionales como modelos de organización social y política, junto al desarrollo del concepto liberaldemócrata de ciudadanía; habría quienes están legitimados para participar públicamente mientras otros quedarían excluidos, lo que queda consagrado con la formalización jurídica que se hace de las personas; mayores y menores de edad, con sus respectivas garantías y deberes. El nombramiento jurídico de la juventud viene acompañado de la creación en las sociedades occidentales de las instituciones necesarias para la segregación y diferenciación de los y las jóvenes respecto a otros grupos generacionales: la escuela y el ejército al principio, el mundo asociativo y el mercado de ocio después. Lo que se espera es la preparación de unos cuerpos aún inestables biológica, psíquica y socialmente, de allí que hablar de juventud supone desde esta interpretación reconocer el carácter de actores sociales a los y las jóvenes y del necesario control social e institucional de las posibilidades de agencia y transformación que poseen.

Por otra parte, la importancia de construir un mapa respecto al campo teórico-político de los estudios sobre juventud en Chile es un tarea pendiente y que cada cierto tiempo surge como preocupación entre los investigadores en juventudes que, cada vez que se reúnen terminan reivindicando la necesidad de sistematizar el acumulado como forma de no estar «partiendo siempre de cero». La importancia de estos factores del desarrollo académico institucional chileno de los estudios sobre juventud radica en que a la fecha no se cuenta con estados del arte sobre juventud como sí existe en otros países (México, Colombia, España). Esta situación es producto de un conjunto de dificultades institucionales y asociativas específicas en que se han desarrollado los investigadores chilenos y los procesos de construcción de conocimiento, lo que se traduce en una fragmentación y discontinuidad entre investigaciones, investigadores e instituciones en los temas juveniles.

Los factores que inciden en esta situación son de distinta naturaleza y alcance, pero el principal ha sido que a pesar de constituir un área frontera en el conocimiento social, debido a la propia complejidad del proceso de constitución del sujeto social sobre el que se investiga, la tendencia general en la investigación sobre juventud en Chile 
ha privilegiado miradas disciplinarias restringidas sin incorporar el necesario tratamiento transdisciplinario. Algo que sin duda está vinculado con la nula inserción institucional de estas perspectivas en el ámbito académico.

Asimismo, ha sido frecuente que algunos investigadores sólo entren coyunturalmente a los estudios de juventud y no permanezcan trabajando en él, lo que ha debilitado la construcción de bases para la formación de todos aquellos que tienen vínculos permanentes con los y las jóvenes; a nivel universitario solo existen cuatro cursos universitarios dedicados al tema (tres en sociología y uno en antropología) y no existe oferta formativa en posgrado, sólo un centro académico universitario (CEJU-UCSH), y sólo una organización no gubernamental dedicada a producir conocimiento relevante sobre jóvenes (CIDPA).

La débil asociatividad de los investigadores y la fragmentación del conocimiento respecto al sujeto juvenil, que sumado a la despreocupación institucional por promover estados generales de la cuestión, constituye otra causa que incide en la debilidad de los procesos de acumulación de conocimiento y generación de nuevos enfoques, temáticas e incluso de estímulo a nuevos investigadores sobre juventud.

Finalmente, el conjunto de investigadores que desde mediados de los años 80 desarrollaron una interesante producción sobre el tema juvenil y que de alguna manera iniciaron el campo de estudios en Chile (Agurto et al., 1985; Undiks et al., 1989; Weinsten, 1988; Valenzuela, 1984; Cottet y Galván, 1993, entre otros) trasladaron sus preocupaciones hacia otros ámbitos de las ciencias sociales y no se generaron, debido a las condiciones institucionales reseñadas previamente, los relevos generacionales necesarios cuando se requiere pensar en la constitución de un área de conocimiento.

Estos procesos que hemos descrito se traducen, en que a) los vacíos en la sistematización del conocimiento acumulado en los estudios de juventud en Chile han sido llenados por los procesos de significación y semantización que despliegan los medios de comunicación como constructores de sentidos y la agenda de la discusión pública sobre juventudes en nuestro país es construida en dichos espacios mediáticos, y b) un proceso que se despliega al interior del propio campo de estudios a través de un permanente adan-evismo de quienes comienzan a investigar sobre juventudes: el mito fundacional de creer ser los primeros en hacerlo.

Reconocidas esas dificultades, me propongo construir una ruta general que, tomando como punto de partida los enfoques sobre ju- 
ventud, posibilite una agenda futura de investigación que permita realizar aquellos estados del arte que tanto se requieren. Para cumplir con dicho propósito contextualizaré: a) las perspectivas fundacionales con que se inician en los años 80 y comienzos de los 90 los estudios de lo juvenil como campo específico de las ciencias sociales, b) para luego presentar los enfoques presentes en la actualidad, y c) presentar una síntesis que desde la perspectiva cultural se hace cargo de algunas tensiones que dificultan una comprensión de las dimensiones políticas en las prácticas juveniles.

\section{EL TRAYECTO HISTÓRICO Y TEÓRICO HEREDADO}

Aún cuando el foco principal de este capítulo remite a los estudios sobre juventud, es imprescindible reconocer la distinción de la juventud como emergencia identitaria y constitución de un actor social (González Cangas, 2002) o como categoría de análisis de las ciencias sociales. En el primer caso, la emergencia de los y las jóvenes como actor social podemos situarlo más claramente a inicios del siglo XX, a partir de las organizaciones artístico-literarias de la época, y a partir de allí la constitución y desarrollo de la Federación de Estudiantes de la Universidad de Chile en 1906 (Aguilera, 2003; González Cangas, 2004; Muñoz, 2007).

Todos estos procesos que hemos caracterizado previamente (Aguilera, 2003) van acompañados de una producción ensayística que se encarga de relevar y visibilizar al emergente actor juvenil en las sociedades latinoamericanas y que se reflejan en la publicación de Ariel de José Enrique Rodó en 1900, los Siete ensayos de la realidad peruana de J. Carlos Mariátegui, o la Carta a la juventud de Vicente Huidobro en 1925. Todo lo cual va reforzando la constitución de una identidad generacional de los sectores juveniles, aunque circunscrita a las élites pequeño-burguesas con acceso a la educación universitaria. De este período se hacen cargo los trabajos de Salazar y Pinto (2002), Vicuña (2001) y los escritos políticos de González Vera y Manuel Rojas (2005), en los cuales se aprecia como los y las jóvenes se incorporan a una sociedad chilena en proceso de cambio social y político, acompañando fundamentalmente aquellas posiciones políticas que apuntan al cambio social democrático y de cierre de la etapa oligárquica.

Mención especial nos merece el trabajo de Vicuña (2001) quien desde la historia cultural reconstruye el periodo de la belle epoque chilena y presenta las tensiones que se producen en el campo genera- 
cional, de género y de sociabilidad en la ciudad de Santiago de principios del siglo XX que se encuentra en pleno proceso de urbanización y desarrollo, y que retrata muy bien a aquella burguesía ilustrada que va aportando con los primeros jóvenes vanguardistas de la cultura como el poeta Vicente Huidobro, que incluso llega a ser proclamado como candidato a la presidencia de Chile en representación de la Juventud. $\mathrm{Y}$ en una suerte de contrapunto, los artículos periodísticos y crónicas escritas por Manuel Rojas y José González Vera nos presentan las furiosas y militantes posturas de los jóvenes anarcosindicalistas que por ese entonces constituían el grupo político con mayor presencia e influencia en el mundo estudiantil y que se expresaba en las revistas universitarias Claridad y La Pluma, ambas vinculadas con la Federación de Estudiantes de la Universidad de Chile (FECH).

En cambio al entender la juventud como categoría de las ciencias sociales se destacan, de acuerdo a González (2004), los trabajos de Aníbal Ponce (1938) Sicología de la adolescencia y Ambición y angustia de los adolescentes o el auge de investigaciones que en el marco de las políticas modernizadoras de la CEPAL en los años 50 reconocen la especificidad del actor juvenil. Pero todas ellas circunscritas en términos no declarados a homologar a la juventud con los estudiantes universitarios, eliminando las especificidades y singularidades juveniles que trascienden este subsector juvenil. De allí que González afirme que «así se puede constatar en América Latina que las indagaciones científicas sobre la reforma universitaria y los procesos políticos continentales y mundiales desde la perspectiva de la juventud universitaria, monopolizaron la mayor parte de la investigación social sobre el actor» (González, 2004:34).

Para los efectos de nuestro punto de partida, podemos afirmar que la categoría social de juventud no comienza a ser estudiada sistemáticamente por las ciencias sociales sino a partir de los años 70. Es paradigmático que la primera publicación al respecto, Juventud chilena. Rebeldía y conformismo de Armand y Michelle Mattelart, data del año 1970. Además, dicho estudio es expresivo de las preocupaciones teóricas de los cientistas sociales de la época: indagar respecto a los procesos de continuidad y cambio social que se encuentran en desarrollo en la sociedad chilena y el papel que juegan en ellos los diversos sujetos sociales. Y aún cuando parte de una dicotomía entre «rebeldes y conformistas»el retrato de la época que nos presenta habla de una heterogeneidad juvenil en sus formas de estar juntos, de proyectar el futuro y posicionarse en el presente; época de una fuerte presencia estudiantil y juvenil en los pro- 
cesos sociales, pero también la emergencia de incipientes agrupaciones juveniles vinculadas alrededor de la industria cultural, época de la reforma universitaria iniciada en la Universidad Católica de Valparaíso en 1967 y que se extiende hacia Santiago en 1968, en paralelo a la difusión del movimiento musical de la «nueva ola» y su expresión mediática en la revista Ritmo que se ocupa fundamentalmente de difundir la versión local y edulcorada en español del rock and roll que había llegado algunos años antes y que había generado una primera camada de solistas y conjuntos musicales de gran impacto y masividad entre amplios sectores de la juventud chilena.

Sin embargo, reiteramos que el trabajo de los Mattelart se trata de una experiencia excepcional, pues no será hasta los años 80 en que comienza una preocupación sistemática por la juventud como actor social específico que requiere, y merece, la atención de las ciencias sociales (Vergara, 2002; Sandoval, 2002). Significativamente, será en contexto de dictadura (1973-1989) que en Chile se desarrolla y consolida la investigación en juventud; en esta etapa las universidades estaban sometidas a un fuerte control social que llegó incluso a la nominación de militares en los cargos de rectores, intelectuales y académicos sufrieron las purgas de los militares y los jóvenes profesionales no tenían espacios para la docencia y la investigación.

En dicho contexto académico y sociopolítico, la mayor parte de estudios sociales, entre los que se incluyen los de juventud, fueron desarrollados por organismos no gubernamentales o por organismos dependientes de la iglesia católica, $\mathrm{y}$ en términos metodológicos predominaron microestudios de carácter cualitativo y exploratorio en que la preocupación temática central de esta etapa era indagar en las acciones juveniles que apuntaban a una reconstrucción del tejido social y político en contexto de dictadura. Asimismo, la juventud popular se constituye en la categoría que emerge en los 80 como resultado de los procesos de transición social (modernización económica y crisis política) que atravesaron la sociedad, «En efecto, la propia existencia de una juventud popular, es posible sociológicamente merced a la extensión de la cobertura de la educación, lo que permite un período de 'moratoria' de roles adultos en los jóvenes de sectores populares, de manera similar a como ocurre en la clase media, aunque con más tiempo libre y menos recursos» (Tsukame, 2000:2).

La preocupación central del periodo está construida alrededor de las formas de acción juvenil en dichos contextos, y de allí que no sea aventurado señalar que el intento referencial por profundizar en el cono- 
cimiento de la juventud lo constituya el texto Juventud chilena. Razones y subversiones (1985), en que podemos reconocer al menos dos lecturas teóricas que permanecerán en la década siguiente: a) una fuerte crítica al concepto de anomia, y su sustitución por matrices teóricas weberianas como en el estudio de Tsukame (1985) quien a partir de las prácticas de consumo de drogas entre jóvenes urbanos de Santiago relativiza las diferencias entre sujeto y objeto (actor/estructura social), iniciando un enfoque comprensivo que será la constante en los estudios de juventud en la década de los 90, y b) una línea de interpretación de la juventud y sus acciones que se realiza desde las categorias de movimiento social emergente y movimiento juvenil popular. La idea central que exponen Agurto, Canales y De la Maza es que las acciones juveniles no son un proceso acabado y con un sentido prístino, más bien señalan que «la acción juvenil de estos años puede entenderse como un intento persistente, nunca triunfante, nunca derrotado, por superar la acción del poder: castigo y exclusión» (Agurto et al., 1985:8).

Este texto, de creación colectiva y que incluye fragmentos de obras de teatro, entrevistas, así como «artículos académicos», en una suerte de performance de las distintas hablas juveniles, necesariamente debe ser leído en relación a un trabajo previo y que avanza en una comprensión diferente de la realidad juvenil chilena; nos referimos a la publicación de La rebelión de los jóvenes. En este trabajo, Valenzuela (1984) señala que las acciones juveniles que desarrollan los jóvenes de Santiago deben ser leídas desde la categoría de «anomia» y agrega que el conflicto normativo entre una estructura social en proceso de modernización que promueve unas metas a conseguir (económicas) y la impotencia de amplios sectores de la juventud chilena (urbana y popular, principalmente) generarían una tensión en los jóvenes y ello explicaría las distintas acciones de protesta o divergencia social en las que incurren los jóvenes. De esta forma, el eje tradición y modernización entran en conflicto generando sectores «dentro de la sociedad» y que generan capitales sociales y culturales que les permiten movilizarse adecuadamente, en tanto otros sectores estarían «fuera de la sociedad», y se caracterizarían por acciones delictivas o de tipo comunitarista.

La idea central expuesta en el texto, y que señala que serían esos desajustes en la estructura normativa provocados por los procesos modernizadores (estructura social) los determinantes de los comportamientos anómicos de los sujetos (actor social), es recuperada en distintos estudios sobre juventud en la actualidad (Guell, 2004; Contreras, 2000; entre otros). En el caso de Contreras (2002), el autor sostiene una hipó- 
tesis que señala que los jóvenes reproducen en su sociabilidad la ideología liberal: la juventud popular estaría en un estado de angustia permanente por no cumplir sus expectativas de vida (pautadas por el neoliberalismo) lo que verificaría el fracaso del itinerario pautado por la modernidad (trabajo-tranquilidad/estabilidad-consumo) con el consiguiente descentramiento de las instituciones encargadas socialmente de verificar este transito desde lo juvenil a lo adulto.

A partir de estos textos emblemáticos de la producción investigativa de la época, podemos concluir que en el campo de los estudios sobre juventud se construyeron dos lecturas teóricas sobre el sujeto juvenil y que se encuentran enfrentadas entre sí: a) la del sujeto parcial, que señala que la juventud se caracterizaría por constituir una particular forma de buscar alternativas dentro de un panorama de escasez de éstas y en dicho marco emerge una «cultura juvenil», entendida en cuanto prácticas juveniles y modos de dar significado a dichas prácticas (Agurto et al., 1985); y b) la del sujeto anómico, que señala que la falta de integración al mundo institucional de la participación lleva al declive de sus formas tradicionales (partidos, organizaciones, formas de acción comunitarias) y a una acción social regida por la adecuación a fines individualistas, todo ello ligado a una disolución de normas y valores fundamentales (Valenzuela, 1984).

\section{LOS ESTUDIOS DE JUVENTUD A PARTIR DE LOS 90}

El fin de la dictadura implicó un reconocimiento explícito de la deuda social con los jóvenes, que según Rodríguez estaría caracterizada por «La situación económica y política existente durante el régimen militar generó una severa exclusión socioeconómica, especialmente en jóvenes de sectores populares urbanos (fuertemente marginados del trabajo, pese a haber alcanzado unos niveles relativamente altos de escolaridad). Dicha situación fue representada o dicha como 'deuda social con los jóvenes' por parte de los actores que en ese momento asumieron la tarea de diseñar políticas de juventud» (2000:92). Y ello tuvo su correlato político en la constitución de los jóvenes como problema, de allí que a partir de la lectura de los ejes temáticos que han predominado en los estudios en juventud en la década pasada, comprobemos que la juventud se constituyó en un cuerpo social a intervenir desde el aparato político mediante la aplicación de políticas sociales.

Esta situación, de orden político e institucional, trajo dos consecuencias inmediatas: a) la proliferación de estudios por encargo de las 
distintas reparticiones públicas con la misión de diagnosticar «las situaciones-problema», y b) un retroceso en la discusión conceptual respecto al sujeto juvenil que se había comenzado a generar a partir de los iniciales investigadores en juventud. Lo que importaba en el contexto del primer gobierno de la concertación por la democracia (19901994) no era problematizar, sino más bien aplicar. Este desplazamiento, orientado desde el propio Estado, se ha consolidado a lo largo del tiempo llegando incluso a definir los temas, las metodologías y por supuesto los enfoques teóricos para abordar la investigación en juventud. ${ }^{1}$ Es a partir de este proceso que podemos explicarnos la preocupación por incluir socialmente a los jóvenes, dando origen a estudios específicos sobre la materia y a políticas concretas por parte del Estado; un ejemplo de esto es que gran parte de los estudios en juventud de los 90 giran en torno a la institución educativa, sea en su variante escolar tradicional (estudiantes secundarios) como en la de formación para el trabajo (juventud popular y en proceso de inserción laboral). Por lo mismo, las orientaciones generales de dichos diagnósticos se orientan más bien a describir antes que a interpretar los sistemas de acción de los jóvenes.

En términos políticos, este periodo se caracteriza por pasar progresivamente de concepciones centradas «en la integración social» a otras centradas en «el control»; ello quiere decir que desde el gobierno asume que existe una «deuda social» con los jóvenes y como consecuencia de ello se procesan algunas demandas, a la vez que existe desconfianza hacia la juventud y su activa participación en la dictadura. La interpretación es que los jóvenes pueden, potencialmente, representar una amenaza para la transición, y paulatinamente se instala como lugar común la supuesta «apatía» de los jóvenes frente a todo lo que signifique participar del proceso democratizador que se iniciaba. «Esta apatía se suponía que tenía directa relación con la inexistencia de espacios para la disputa y negociación por el sentido, de un orden social que se considera preestablecido, y que opera según flujos de integración y exclusión. La apatía juvenil era leída como una crítica impotente al sistema democrático que se estrenaba en la transición» (Tsukame, 2000:5).

Es así como desde este marco comprensivo general se desprenden los ejes temáticos que han atravesado las investigaciones sobre jóve-

1 Posteriormente podremos apreciar cómo esta situación se traduce en la investigación en el campo educativo, a partir de la relación entre culturas juveniles y culturas escolares. 
nes en Chile durante los años 90 hasta fines de siglo, y que posteriormente nos permitirán presentar los enfoques predominantes en los estudios sobre juventud:

Joven dañado: el énfasis está puesto en aquellas personas jóvenes que pudieran presentar «problemas» de integración social producto de accidentes biográficos en sus trayectorias vitales. Hablamos, fundamentalmente, de la juventud empobrecida que no ha contado con unas condiciones de vida diga que les permitan un desarrollo integral (Oyarzún, 2001).

Conversación juvenil: se refiere a la posibilidad de construir sentidos sociales más amplios, a partir de la pluralidad de voces presentes en el mundo juvenil. Esta concepción nace a partir de experiencias de intervención en programas de desarrollo juvenil por parte de algunas ONG'S. (Cottet y Galván, 1993).

Acción juvenil: el énfasis de este eje investigativo se encuentra en considerar la dimensión de acción y actores que asumen o no las personas jóvenes. Surgen así nociones como: juventud integrada, juventud en conflicto, juventud dominada (Undiks et al., 1990; Duarte, 1994).

Sociabilidad y socialidad juvenil: aquí ubicamos el surgimiento de estudios respecto a las modalidades juveniles de estar juntos y que son construidas a partir de la espectacularidad mediática; barras de fútbol, estilos juveniles y los consumos en contexto de carrete juvenil. (Matus, 2000; Zarzuri y Ganter, 1998).

Violencia juvenil: aquí encontramos estudios, la mayoría por encargo de servicios públicos, que intentan investigar los procesos de violencia juvenil en los sectores populares, pero que progresivamente se van centrando en los espacios escolares (INJUV, 1998).

\section{ENFOQUES ACTUALES SOBRE JUVENTUD}

Para concluir nuestro recorrido por los estudios sobre juventud en Chile, proponemos acceder al campo investigativo a partir del reconocimiento de tres grandes concepciones sobre la juventud, que como todo intento de esquematizar está sujeto a los criterios de organización que se adopten. En este caso, se ha procedido por una categorización más amplia de enfoques reconocibles que algunas de las construidas por otros autores (Sandoval, 2002; Duarte, 2005) y proponemos que en su interior realicemos la distinción de perspectivas y trayectorias específicas: 
Enfoque sociodemográfico: aquí encontramos perspectivas que no cuestionan mayormente la categoría juventud, y que asumen la convención de definirla a partir de unos determinados rangos de edad (demografía), así como otras que la definen como un proceso de aprendizaje social e internalización del sistema normativo (moratoria). El foco de estas perspectivas se centra en el tránsito hacia la integración social, desde una perspectiva funcional en el marco de las políticas públicas.

Enfoque psicológico: aquí encontramos perspectivas que están más cercanas a las corrientes de la psicología del desarrollo y que por tanto se preocupan de los procesos de maduración psicobiológica, y otras perspectivas que avanzan hacia el análisis de aspectos identitarios y sociales. El foco de estas perspectivas se centra en la constitución de la identidad individual y colectiva.

Enfoque sociocultural: en esta categoría ubicamos perspectivas que enfatizan en el carácter socialmente construido, y por tanto relacional del concepto de juventud a partir de su articulación con miradas generacionales, miradas de enfoque de derechos, de la acción colectiva, y de la cultura y sociabilidad juvenil.

Estos tres grandes enfoques necesariamente debieran ser leídos, a su vez, como enmarcados por una tensión epistemológica respecto al tipo de relación que se construye entre el investigador y el mundo juvenil, y que atraviesa muchos de los enfoques anteriormente descritos. Esta tensión se traduce en dos orientaciones claramente reconocibles:

Orientación universalista; esta orientación se caracteriza por asumir la existencia de un patrón unitario a partir del cual se construiría la noción de juventud. Dentro de esta orientación ubicamos el adultocentrismo como una matriz generativa que supone la preeminencia del adulto por sobre los niños, jóvenes y viejos, y desde el cual se asignan atributos, significados y explicaciones a las acciones de quienes no lo son.

Orientación relativista; esta orientación se caracteriza por desconocer la existencia de algún tipo de referencialidad externa acerca de lo que sería la juventud. Dentro de esta orientación reconocemos la existencia de una matriz generativa de tipo identitaria que supone la imposibilidad de generar comprensiones totales sobre el sujeto juvenil y por tanto hay que detenerse en sus especificidades evitando las generalizaciones. 
Por la importancia que tiene para nuestro estudio sobre la acción colectiva, profundizaremos en esta segunda orientación a partir de una crítica a los usos y lecturas del concepto de «tribu urbana» pero con el objeto de intentar desde el reconocimiento de las particularidades juveniles no perder el horizonte comprensivo universal, generando una perspectiva epistemológica que nos aproxime desde la sospecha, pero también desde el encantamiento (Reguillo, 2000).

\section{TRIBUS URBANAS E IMPOSIBILIDADES POLÍTICAS}

Toda identidad es fundamentalmente ambigua señala Balibar (2006), y lo que quiero destacar con la ayuda de este filósofo francés es que no podemos suponer que un conjunto de atributos culturales que evidencie (exhiba) un individuo o una colectividad puede suponer automáticamente la homogeneidad cultural del grupo y la de los propios individuos. Entre otras cosas, porque no existe una identidad dada sino identificaciones (Balibar, 2006; Maffesoli, 1990) que siempre dependen de las contingencias, de los otros, de las circunstancias materiales que nos rodean; se trata de una construcción eminentemente relacional.

Sin embargo, no creo que esta forma hegemónica de comprender las identidades como un todo homogéneo sea fácilmente desalojada de nuestros contextos culturales y de nuestras expresiones cotidianas. Pero así como en un momento de nuestra historia los universales cerraron toda posibilidad de reivindicación identitaria no inscrita en esos registros, hoy paradójicamente asistimos a una re-instalación de «particulares» pero que niegan, en su afirmación, la posibilidad de tensión y jerarquización en las construcciones identitarias individuales y colectivas al interior de las grupalidades; «establecer una jerarquía de referencias comunitarias no es absorber su diversidad en la uniformidad de una sola pertenencia 'totalitaria'; por el contrario es constituir aquello que gracias a un nuevo préstamo tomado del léxico de Gramsci podemos denominar hegemonía en el seno mismo de la ideología» (Balibar, 2006:73). Esta cuestión es importante porque aquí encontramos un vínculo con la orientación epistemológica relativista que tensiona a muchos de los investigadores en juventud y centrados en el análisis de aquellas prácticas espectaculares que hablarían de la multiplicidad de sujetos juveniles; las tribus (Zarzuri y Ganter, 1999; Silva, 1999; Matus, 2000).

Una tribu, en su definición antropológica, remite a una forma de organización sociopolítica carente de estratificación social y de go- 
bierno centralizado y cuya constitución recae en lazos familiares sanguíneos y políticos, el compartir territorios y una lengua (Kottak, 2007), o en la formulación de Sahlins (1972) para quien la tribu es, como la nación en su sentido antiguo, un grupo de gentes de origen y costumbres comunes, en posesión y dominio de un territorio extensivo propio. Si esas definiciones las utilizáramos para analizar hoy en día los agrupamientos humanos contemporáneos tendríamos serías dificultades para distinguir quiénes o cuáles grupos constituirían tribus y cuáles no, por lo tanto se instala la sospecha; ¿cuáles son los criterios para construir unas prácticas culturales como tribales, mientras que otras que satisfaciendo las condiciones de inclusión en el concepto quedan fuera?

El ex presidente Ricardo Lagos señaló hace muy poco tiempo en declaraciones a la prensa (2008); «No estoy dispuesto para una tribu en particular», en alusión a la definición de los candidatos presidenciales de la coalición de gobierno. Más allá de la corrección o coloquialismos en el uso del concepto, es interesante observar las reacciones del mundo político quienes coincidieron en que se trataba de una afirmación «poco feliz», una palabra «desafortunada». Nuevamente surge la pregunta de ¿qué es lo que entienden nuestros políticos como tribu? Lo que no deja lugar a dudas es que el uso del calificativo los agravió profundamente, pues se trataría de una forma peyorativa de nombrar los pequeños agrupamientos que coexisten al interior de los partidos y las coaliciones políticas. Sostengo entonces que aquí se abre un nuevo nudo en lo que respecta a las «tribunas urbanas», más aún si aceptamos dos supuestos básicos que constituyen la definición del concepto de tribalismo que nos propone Maffesoli (1990), a saber:

Si la modernidad se caracteriza por señalar residencia — pertenecemos a una profesión, un sexo, una ideología, una clase; en pocas palabras, cada cual tiene una identidad y una dirección, cuyo conjunto determina un social racional, mecánico y finalizado - resulta curioso constatar que la socialidad contemporánea es mucho más confusa, heterogénea y móvil. [...] Si las tribus se han afirmado en torno al hermetismo (gusto del secreto, uniformidad en el vestir y en los modos de vida), las personas (persona-máscara) que las constituyen circulan, por su parte, de un grupo a otro con el objeto de poner en práctica la pluralidad de sus máscaras (Maffesoli, 1990:42).

Si la movilidad y la pluralidad definen a los agrupamientos tribales reseñados por Maffesoli, ¿cómo entender entonces el uso social y académico de este mismo concepto que remite, en un permanente proceso de 
reificación, a la unicidad de los atributos de las tribus, a la homogeneidad en sus integrantes, a una nueva universalización ahora sustentada en la diferenciación individualizada, y a una concepción estática de concebir los atributos culturales por parte de individuos y grupo?

Esto nos obliga necesariamente a ubicar el desarrollo y masificación de esta conceptualización en el marco de lo que el Kosselleck (1993) ha denominado «histórica» es decir, que los conceptos se encuentran ubicados y producidos en contextos específicos constituyendo un campo semántico que se alimenta mutuamente, así como nos exige recuperar el valor relacional (con el contexto, y con otros textos) que tienen los conceptos con los cuales trabajamos; "se pueden estudiar los fenómenos sociales como sistemas de significación, esto es, como sistemas de diferencias. Para estas tradiciones constructivistas y profundamente anti-esencialistas, el valor de un término discursivo no es intrínseco sino puramente relacional y, por ende, extrínseco: surge como resultado contingente de prácticas articulatorias, lo cual quiere decir que el valor de un término puede ser modificado a través de otros modos de articulación» (Arditi, 2000:8).

$\mathrm{Y}$ es que el problema de la diversidad cultural y la multiplicación de referentes identitarios, así como hace posible (y visibiliza) la existencia de distintas formas de vida que se encuentran presentes en una sociedad, tiene su propio reverso al momento de pensar en articular la acción colectiva porque esa misma diversidad opera como fronteras que impiden la comunicación entre los jóvenes. Esto es lo que en los estudios culturales y de los movimientos sociales se ha denominado «políticas de la identidad»; «este auge de las diferencias y del modo de acción política basada en la identidad redefinió las coordenadas del pensamiento progresista de fin de siglo [...] hoy en día hay suficientes indicios que muestran que la política de la identidad tiende a cerrarse en un esquema particularista donde eventualmente todo, o casi todo lo que no es enunciado desde un grupo particular, puede ser visto como un agravio para sus integrantes. Se trata de la exaltación de una modalidad acotada del 'nosotros' y la devaluación de otra más amplia» (Arditi, 2000:9).

Las implicaciones de estas cuestiones son centrales para el estudio de las juventudes en tanto la propia relacionalidad del concepto nos obliga no sólo analizar lo que ocurre entre pares, sino que también las relaciones intergeneracionales y no asumir a priori que se trata de conjuntos homogéneos ni unos ni otros. Sostengo además que si nuestras concepciones de las identidades y las comunidades siguen invisibilizando las tensiones que acabo de reseñar, las posibilidades políticas y 
emancipatorias están seriamente delimitadas. No es menor recordar, finalmente, que toda noción de ciudadanía contiene una «negación antropológica de un otro» que no califica, con lo cual se generan «categorías de la presencia» de aquellos que son prescindibles, con lo cual se debilitan las posibilidades de construcción del vínculo civil, fundamento de la confianza social que queda reducida a unos mínimos y que redefinen finalmente las posibilidades de reciprocidad intersubjetiva.

SANTIAGO (CHILE), JUNIO DE 2009

RECIBIDO: JULIO 2009

ACEPTADO: AGOSTO 2009

\section{REFERENCIAS BIBLIOGRÁFICAS}

Aguilera, O. (2008): «Movidas, movilizaciones y movimientos. Cultura política y políticas de las culturas juveniles en el Chile de hoy». Tesis Doctoral. Barcelona: Universitat Autónoma de Barcelona. (2003): «Tan jóvenes, tan viejos. Los movimientos juveniles en el Chile de hoy». Documento Café Diálogos. Santiago: Instituto Nacional de la Juventud.

Agurto, I; M. CANALES et al. (1985): Juventud chilena. Razones y subversiones. Santiago: ECO/FOLICO/SEPADE.

ARDITI, B. (2002): El reverso de la diferencia. Identidad y política. Caracas: Editorial Nueva Sociedad.

BALARDINI, S. (2002): Córdoba, cordobazo y después. Mutaciones del movimiento juvenil en Argentina. En FEIXA, SAURA y COSTA: Movimientos juveniles. De la globalización a la antiglobalización. Barcelona: Ariel Social.

Balibar, E. (2006) Violencias, identidades y civilidad. Para una cultura política global. Barcelona: Gedisa.

CONTRERAS, R. (2002): «Las imágenes del paraíso: juventud popular, liberalismo y sociabilidad en Chile». Última Década $\mathrm{N}^{\circ} 16$. Viña del Mar: Ediciones CIDPA.

- (2000): «Fragmentación social y sociabilidad: la juventud como síntoma de la sociedad neoliberal». Tesis de Maestría. Santiago: ILADES.

COTTET, P. y L. GALVÁN (1993): Jóvenes: una conversación social por cambiar. Santiago: ECO, Educación y Comunicaciones.

DUARTE, C. (2005): «Trayectorias en la construcción de una sociología de lo juvenil». Persona y Sociedad, Vol. XIX, N³. Santiago: Universidad Alberto Hurtado.

(1994): Juventud popular: el rollo entre ser lo que queremos, o ser lo que nos imponen. Santiago: LOM Ediciones. 
FALETTO, E. (1986): «La juventud como movimiento social en América Latina». Revista de la CEPAL No29. Santiago: CEPAL.

FEIXA, C. (1998): De jóvenes, bandas y tribus. Barcelona: Ariel.

GONZÁLEZ, Y. (2004): «Óxidos de identidad: memoria y juventud rural en el sur de Chile 1935-2003». Tesis Doctoral en Antropología. Barcelona: UAB. (2002): «Que los viejos se vayan a sus casas. Juventud y vanguardias en Chile y América Latina». En FEIXA, SAURA y COSTA: Movimientos juveniles. De la globalización a la antiglobalización. Barcelona: Ariel Social.

GUELL, P. (2004): Transformaciones socioculturales. Santiago: INJUV.

HALL, S. (1977): Los hippies: una contracultura. Barcelona: Anagrama.

— y T. JEFFERSON (editores) (1983): Resistance Through Rituals. Youth Subcultures in post-war Britain. London: Hutchinson.

HALL, S. G. (1915) [1904]: Adolescence: Its Psychology and its relations to Psysiology, Sociology, Sex, Crime, Religion and Education. New York: Appleton Century Crofts.

HedBige, D. (2003): Subcultura. El significado del estilo. Barcelona: Paidós. INJUV (1998): Pandillas juveniles urbanas. Santiago: INJUV.

Koselleck, R. (1993): Futuro pasado. Para una semántica de los tiempos históricos. Barcelona: Paidós.

KOTTAK, C. (2007): Introducción a la antropología cultural. Espejo para la humanidad. Madrid McGraw Hill (quinta edición).

MAFFESOLI, M. (1990): El tiempo de las tribus. El declive del individualismo en la sociedad de masas. Barcelona: Icaria.

MATUS, C. (2000): «Tribus urbanas: entre ritos y consumos. El caso de la discoteque Blondie». Última Década $\mathrm{N}^{\circ} 13$. Viña del Mar: Ediciones CIDPA.

MEAD, M. (1985): Adolescencia, sexo y cultura en Samoa. Barcelona: Planeta.

_- (1977) Cultura y compromiso. El mensaje a la nueva generación. Barcelona: Granica.

MuÑoz, V. (2006): ACU: rescatando el asombro. Santiago: Editorial La Calabaza del Diablo.

OYARZÚN, A. et al. (2001): Entre jóvenes re-productores y jóvenes coconstructores: sentidos de la integración en la cultura escolar. Viña del Mar: Ediciones CIDPA.

REguillo, R (2000): «Entre la insubmissió i l'obediència. Cossos juvenils, politiques d'identidat». En Joves entre dos mons. Moviments juvenils a Europa i a L'América Llatina. Barcelona: Secretaría General de Joventut. (1995): En la calle otra vez. Las bandas: identidad urbana y usos de la comunicación. Guadalajara: ITESO.

RodRÍGUEZ, M. (2000): «Reflexión sobre la experiencia de política de juventud en Chile». Última Década $\mathrm{N}^{\circ} 12$. Viña del Mar: Ediciones CIDPA.

RoJAS, M. y S. GONZÁLEZ (2005): Letras anarquistas. Selección a cargo de Carmen Soria. Santiago: Planeta. 
ROMANí, O. (1989): «Proceso de modernización, cultura juvenil y drogas». En F. RODRÍGUEZ (editor): Comunicación y lenguaje juvenil. Madrid: Fundamentos.

SAHLINS, M. (1972): Las sociedades tribales. Barcelona: Labor.

SAlAZAR, G. y J. PINTO (2002): Historia de Chile. V. 5. Infancia y juventud. Santiago: LOM Ediciones.

SANDOVAL, M. (2002): Jóvenes del siglo XXI: sujetos y actores en una sociedad en cambio. Santiago: UCSH.

SiLVA, J. (1999): «Ni héroes ni malvados, sólo jóvenes. Claves para iluminar la conversación sobre juventudes de los noventa». Última Década $\mathrm{N}^{\circ} 11$. Viña del Mar: Ediciones CIDPA.

Thrasher, F. M. (1963) [1926]: The Gang. A Study of 1313 gangs in Chicago. Chicago: University of Chicago Press.

TsuKame, A. (2000): «Discursos sobre los jóvenes. Apuntes para iniciar proyecto de investigación Fondecyt». Santiago (policopiado). (1985): «Drogas y sentido de la identidad juvenil hoy». En AGURTO, CANALES et al.: Juventud chilena. Razones y subversiones. Santiago: ECO/FOLICO/SEPADE.

Urteaga, M. (2000): «Formas de agregación juvenil». En J. A. PÉREZ IsLAS (coordinador): Jóvenes: una evaluación del conocimiento. La investigación sobre juventud en México 1986-1999. México: Instituto Mexicano de la Juventud.

UNDIKS, A. (1990): Juventud urbana y exclusión social. Buenos Aires: Ediciones Humanitas y FOLICO.

VALENZUELA, E. (1984): La rebelión de los jóvenes. Santiago: SUR Ediciones.

VALENZUELA, J. M. (2002): «De los pachucos a los cholos. Movimientos juveniles en la frontera México-Estados Unidos». En FEIXA, Molina y Alsinet: Movimientos juveniles en América Latina. Pachucos, malandros, punketas. Barcelona: Ariel Social.

Vergara, A. (2002) (editora): Cartografias de la juventud. Revista de Psicología y Ciencias Humanas. Santiago: Universidad Diego Portales.

VICUÑA, M. (2001): La belle époque chilena. Santiago: Sudamericana.

WeINSTEIN, J. (1988): Los jóvenes pobladores en las protestas nacionales (1983-1984). Una visión sociopolítica. Santiago: CIDE.

Whyte, W. F. (1972) [1943]: Street Corner Society. Chicago: University of Chicago Press.

ZARZURI, R. (2000): «Notas para una aproximación teórica a nuevas culturas juveniles: tribus urbanas». Última Década $\mathrm{N}^{\circ} 13$. Viña del Mar: Ediciones CIDPA.

y R. GANTER (1999): «Tribus urbanas: por el devenir de nuevas sociabilidades juveniles». Revista Perspectivas $\mathrm{N}^{\mathrm{o}}$ 8. Santiago: Universidad Católica Silva Henríquez. 